\title{
A Group Theoretical Formalization of Contact Motion
}

\author{
Yanxi Liu \\ The Robotics Institute, Carnegie Mellon University, Pittsburgh 15213, USA, \\ yanxiocs.cmu. edu, \\ http://www.cs.cmu . edu/ yanxi/www/home.html
}

\begin{abstract}
The work reported in this paper applies group theory to characterize local surface contact among solids, and makes such a formalization computationally tractable. This paper serves three purposes: (1) to report a new treatment of contacting surfaces as oriented sets and to verify the consistency of their corresponding symmetry groups in a group theoretical formalization framework; (2) to give a concise summary of this group theoretical approach, from theory, to algorithms, to applications; (3) to pinpoint some unsolved problems and possible future directions.
\end{abstract}

\section{Introduction}

Contact motion analysis of rigid bodies is one of the most useful yet difficult topics in robotics, design, assembly planning and manufacturing. Increasing level of automation demands more effective computational method for representing and reasoning about contacts, and transforming high-level specifications to lowlevel executable commands. Admittedly, contact analysis is "a computational bottleneck in mechanical design", "it is especially challenging for curved parts with multiple, changing contacts" $[5,6]$. The current state of art in this area has left much to be desired. The input to almost all the reported automatic assembly planning systems, such as [24,25, 16,4], is one-static-state of the final assembly configuration regardless the assembly is meant to be rigid or articulated. Most work in contact analysis is dealing with planar surfaces $[22,17,7]$. While the most impressive work on higher pairs ${ }^{1}$ analysis and simulations [5, 21,2] still need human intervention, there exist no formal theory and algorithms for contact analysis of rigid bodies in general.

Hervè [3] and Popplestone [19] are among the few pioneers who contributed to the group theoretical formalization of mechanical engineering practice. Hervé has introduced a rational classification of mechanisms by applying the theory of continuous groups. Since each lower-pair allows a set of relative motions of two coupled bodies, these motions can be regarded as subgroups of $\mathcal{E}^{+}$. The

\footnotetext{
${ }^{1}$ Lower pairs are mechanical joints that have surface to surface contact (area contact). otherwise they are called Higher pairs (line/curve/point contact) in mechanical engineering.
} 
number of independent variables required to define the relative position of two coupled links is referred to as their degree of freedom, which can be extended to a subgroup of $\mathcal{E}^{+}$in 3 -space, the corresponding concept being that of dimension. In [3], Hervè gave a table (Table III) listing the intersection and composition of continuous subgroups of the proper Euclidean group. Only pairs of continuous subgroups are considered. In [19] Popplestone relates robotics and group theory, in particular he pointed out that 1) the symmetry group of a feature (of a solid) is perhaps more important than that of the whole solid, and 2) not only continuous groups (as treated in [3]) but also finite and discrete groups should be handled.

In Liu's Ph.D. thesis $[9,11]$, the group theoretical formalization of surface contact among solids was further extended and solidified. The novelties in her work include, (1) both finite and continuous groups are treated under a uniform general formalization; (2) a simple geometric algorithm for combining different symmetry groups of local contacting surfaces, to determine relative motions of the contacting solids, is developed and implemented with proven tractability. (3) the approach carries through: starting from CAD models of solids, followed by automatic determination of relative locations/motions of multi-contactassembly-parts, to the generation of assembly procedures for robots [12-14]. Liu's notations and algorithms can deal with symmetry groups which is itself a semidirect product of a continuous group and a discrete group, thus providing the power of reasoning about both degrees of freedom and combinatoric configurations of contacting solids simultaneously. A list of some important canonical subgroups of $\mathcal{E}^{+}$treated in [9] is given in Table 1. Further development after [9] includes,

Table 1. Some important subgroups of $\mathcal{E}^{+}$

\begin{tabular}{|c|c|}
\hline $\begin{array}{c}\text { Canonical } \\
\text { Groups }\end{array}$ & Definition \\
\hline $\mathcal{G}_{\text {id }}$ & $\{1\}$ \\
\hline $\mathcal{T}^{1}$ & $\operatorname{gp}\{\operatorname{trans}(0,0, z) \mid z \in \Re\}$ \\
\hline $\mathcal{T}^{2}$ & $\operatorname{gp}\{\operatorname{trans}(x, y, 0) \mid x, y \in \Re\}$ \\
\hline $\mathcal{T}^{3}$ & $\operatorname{gp}\{\operatorname{trans}(x, y, z) \mid x, y, z \in \Re\}$ \\
\hline$S O(3)$ & $\operatorname{gp}\{\operatorname{rot}(\mathbf{i}, \theta) \operatorname{rot}(\mathbf{j}, \sigma) \operatorname{rot}(\mathrm{k}, \phi) \mid \theta, \sigma, \phi \in \Re\}$ \\
\hline$S O(2)$ & $\operatorname{gp}\{\operatorname{rot}(\mathbf{k}, \theta) \mid \theta \in \Re\}$ \\
\hline$O(2)$ & $\operatorname{gp}\{\operatorname{rot}(\mathrm{k}, \theta) \operatorname{rot}(\mathbf{i}, n \pi) \mid \theta \in \Re, n \in \mathcal{N}\}$ \\
\hline $\mathcal{G}_{c y l}$ & $\operatorname{gp}\{\operatorname{trans}(0,0, z) \operatorname{rot}(\mathbf{k}, \theta) \operatorname{rot}(\mathbf{i}, n \pi) \mid n \in \mathcal{N}, \theta, z \in \Re\}$ \\
\hline $\mathcal{G}_{\text {dir_cyl }}$ & $\operatorname{gp}\{\operatorname{trans}(0,0, z) \operatorname{rot}(\mathbf{k}, \theta) \mid z, \theta \in \Re\}$ \\
\hline $\mathcal{G}_{\text {plane }}$ & $\operatorname{gp}\{\operatorname{trans}(x, y, 0) \operatorname{rot}(\mathbf{k}, \theta) \mid x, y, \theta \in \Re\}$ \\
\hline $\mathcal{G}_{\text {screw }}(p)$ & $\operatorname{gp}\{\operatorname{trans}(0,0, z) \operatorname{rot}(\mathbf{k}, 2 z \pi / p) \mid z \in \Re\}$ \\
\hline $\mathcal{G}_{T_{1} C_{2}}$ & $\operatorname{gp}\{\operatorname{trans}(0,0, z) \operatorname{rot}(\mathbf{i}, n \pi) \mid n \in \mathcal{N}, z \in \Re\}$ \\
\hline$D_{2 n}$ & $\operatorname{gp}\{\operatorname{rot}(\mathbf{k}, 2 \pi / n) \operatorname{rot}(\mathbf{i}, m \pi) \mid m, n \in \mathcal{N}\}$ \\
\hline$C_{n}$ & $\operatorname{gp}\{\operatorname{rot}(\mathbf{k}, 2 \pi / n) \mid n \in \mathcal{N}\}$ \\
\hline $\mathcal{E}^{+}$ & $\operatorname{gp}\{\operatorname{trans}(x, y, z) \operatorname{rot}(\mathbf{i}, \theta) \operatorname{rot}(\mathbf{j}, \sigma) \operatorname{rot}(\mathbf{k}, \phi) \mid x, y, z, \theta, \sigma, \phi \in \Re\}$ \\
\hline
\end{tabular}


transforming high-level spatial relation descriptions to low-level compliant motions [15]; treating each oriented surface on a solid as a primitive feature; and obtaining an explicit expression for general contact relationships in terms of the symmetry groups of the contacting surfaces. The last two parts are described in this paper. We are now ready to go beyond surface contact, to venture into more general algebraic surfaces, higher pairs and group products. Different from the study of solids in local contact, e.g., [8,18], our aim is to have a precise and complete description of the intended, possibly articulated, final assembly configuration of solids where each part usually has multiple contacts with the rest of the assembly; and our approach is algebraic in nature. Also different from [20, 23 ] in that a group theoretical formalism is embedded in a concise representation of contact motions not involving extensive algebraic equation manipulation.

\section{Basic Group Theoretical Formalism of Contact Analysis}

Since contacts among solids happen via the contacts of the surfaces of the solids, the representation and characterization of each surface constitutes the foundation of any formalization for solid contacts.

\subsection{Oriented Surface and Its symmetry Group}

The surfaces which we have treated mathematically as subsets of $\Re^{3}[9,11]$ have no intrinsic inside and outside. To remedy this we introduce the concept of oriented features by defining a set of outward-pointing normal vectors for each surface point of a solid. The polynomial used to express an algebraic surface implicitly defines such normal vectors. Let $\mathcal{S}^{2}$ be the unit sphere at the origin embedded in $\Re^{3}$, each point of $\mathcal{S}^{2}$ corresponds to a unit vector in $\Re^{3}$.

Definition 2.1.1 An oriented primitive feature $F=(S, \rho)$ of a solid $M$ is an oriented surface where

1) $S \subset \Re^{3}$ is a connected, irreducible ${ }^{2}$ and continuous algebraic surface which partially or completely coincides with one or more finite oriented faces of $M$;

2) $\rho \subset S \times \mathcal{S}^{2}$ is a continuous relation. For each $s \in S$ if $s$ is a non-singular point of surface $S$ (p.78 [1]) then $v \in \mathcal{S}^{2}$ is one of two opposing normals of the tangent plane at point $s$ such that $(s, v) \in \rho$; if $s$ is a singular point of $S$ (e.g. at the apex of a cone) then, for all $v$, where $v \in \mathcal{S}^{2}$ is the limit of the orientations of its neighborhood, $(s, v) \in \rho$.

3) For all $s \in M,(s, v) \in \rho, v$ points away from $M$.

Intuitively speaking, a feature is composed of both "skin", $S$, and "hair", the set of normal vectors which correspond to the points on $\mathcal{S}^{2}$. Each element of

${ }^{2}$ Here irreducible implies that a primitive feature cannot be composed of any other more basic surfaces. 
relation $\rho$ is a correspondence between a point on $S$ and a vector on $\mathcal{S}^{2}$. Note, there may be more than one 'normal vector' at one point of a surface, e.g. at the apex of a conic shaped surface.

Let $\mathcal{E}^{+}$be the proper Euclidean group which contains all the rotations and translations in $\Re^{3}$, and $\mathrm{T}^{3}$ be the maximum translation subgroup of $\mathcal{E}^{+}$.

Definition 2.1.2 Any isometry $g=\operatorname{tr}$ of $\mathcal{E}^{+}, t \in \mathbf{T}^{3}, r \in S O(3)$ acts on $\rho$ in such a way that $(s, v) \in \rho \Leftrightarrow(g s, r v) \in g * \rho$.

Definition 2.1.3 An isometry $g \in \mathcal{E}^{+}$is a proper symmetry of a feature $F=(S, \rho)$ if and only if $g(S)=S$ and $g * \rho=\rho$.

Note, there is an extra demand on a symmetry for an oriented feature - it has to preserve the orientations $\rho$ of the feature as well as the point set $S$. Since orientations are points on $\mathcal{S}^{2}$, symmetries of an oriented feature have to keep two sets of points in $\Re^{3}$ setwise invariant. One can prove ${ }^{3}$ that the symmetries for an oriented surface form a group.

\subsection{Multiple Contacts: Compound Surface Features and Their Symmetry Groups}

An assembly or mechanism is a manifestation of multiple surface interactions of its subparts, albeit the physical property of each individual part (rigid or deformable) or the nature of the contact (static or articulated). Thus the representation of an assembly or mechanism is reduced to how to specify a set of contact constraints which dictate the configuration of a set of solids. Given two solids $B_{1}$ and $B_{2}$ in contact via surfaces $F_{1}$ and $F_{2}$ respectively, the relative motions of $B_{2}$ respect to $B_{1}$ can be expressed as:

$$
l_{1}^{-1} l_{2} \in f_{1} G_{1} G_{2} f_{2}^{-1}
$$

where $l_{1}^{-1} l_{2}$ is the relative position of solid 2 w.r.t. solid $1, G_{1}, G_{2}$ are symmetry groups of $F_{1}$ and $F_{2}$ respectively, $l_{1}, l_{2}$ specify the locations of solids $B_{1}, B_{2}$ in the world coordinate system and $f_{1}$ and $f_{2}$ specify the locations of $F_{1}, F_{2}$ in their respective body coordinates. A more specific form for the relative positions of two solids under $n$ surface contacts (two contacting surfaces coincide):

$$
l_{1}^{-1} l_{2} \in f_{1} G f_{2}^{-1}
$$

has shown clearly that the possible motions of a solid or a subassembly $S$ in an assembly are described precisely by the symmetry group $G$ of the multiple contacting oriented surfaces of $S$. Note, $G$ here is usually not the symmetry group of the whole solid/subassembly $S$ but the symmetry group of those surfaces of $S$ that are in contact with other solids. If $G$ is an identity group, i.e. $l_{1}^{-1} l_{2}=f_{1} f_{2}^{-1}$ gives a fixed position for $S$. If $G$ is a finite rotation group, then $f_{1} G f_{2}^{-1}$ contains

${ }^{3}$ Due to space limit, we only give results without proofs. Interested readers can find details in the references listed. 
a finite number of positions reflecting the existence of discrete symmetries in the collection of contacting surfaces. If $G$ is a continuous group then there exists relative continuous motions between $S$ and the rest of the assembly. It is then the degrees of freedom of such a configuration can be spoken of.

Let us first give a denotation for such a collection of contacting surfaces from a single solid, and then determine what effect the symmetry group of this surface-set will impose on the relative motions of contacting solids.

Definition 2.2.1 A compound feature $F=(S, \rho)$ of primitive features $F_{1}=$ $\left(S_{1}, \rho_{1}\right), \ldots, F_{n}=\left(S_{n}, \rho_{n}\right)$, is defined to be $S=S_{1} \cup \ldots \cup S_{n}$ and $\rho=\rho_{1} \cup \ldots \cup \rho_{n}$.

Pairwise Relationship of Oriented Features In order to determine the symmetry group of a compound feature systematically, we start with the simplest compound feature - a compound feature composed of only one pair of primitive features. See Figure 1(a), 1(b) and Figure 1(c) for examples of these simple compound features ( Note that only a finite face on each primitive feature is drawn).

Given a pair of primitive features, what kind of relationship holds between the two features and what is the effect of such a relationship in terms of determining their symmetry group? The following definition gives a characterization of four relationships between a pair of primitive features:

Definition 2.2.2 Two oriented primitive features $F_{1}=\left(S_{1}, \rho_{1}\right), F_{2}=\left(S_{2}, \rho_{2}\right)$ are said to be

- Distinct: if for any open subsets $S_{1}^{\prime} \subset S_{1}, S_{2}^{\prime} \subset S_{2}$, no $g=t r \in \mathcal{E}^{+}$exists such that $g\left(S_{1}^{\prime}\right) \subset S_{2}$ or $g\left(S_{2}^{\prime}\right) \subset S_{1}$. See Figure 1(a) for an example of a pair of distinct features $F_{1}, F_{2}$.

- 1-congruent: if there exists at least one $g \in \mathcal{E}^{+}$such that $g\left(S_{1}\right)=S_{2}$ and $g * \rho_{1}=\rho_{2}$, but for all such $g, g\left(S_{2}\right) \neq S_{1}$. For an example see Figure $1(\mathrm{~b})$. Another example is two parallel planar surfaces with normal vectors pointing in the same direction.

- 2-congruent: if there exists $g_{c} \in \mathcal{E}^{+}$such that $g_{c}\left(S_{1}\right)=S_{2}, g_{c}\left(S_{2}\right)=S_{1}, g_{c} *$ $\rho_{1}=\rho_{2}$ and $g_{c} * \rho_{2}=\rho_{1}$. For an example, consider two parallel cylindrical surfaces having the same radius and normal vectors pointing away from their center lines, as in Figure 1(c). Also, two parallel planar surfaces with normal vectors pointing to the opposite directions serve as examples of a pair of 2-congruent features.

- Complementary: if there exists $g \in \mathcal{E}^{+}$such that $g\left(S_{1}\right)=S_{2}$ and $g *$ $\rho_{1}=\Leftrightarrow \rho_{2}$ where $\Leftrightarrow \rho_{2}=\left\{(s, \Leftrightarrow v) \mid(s, v) \in \rho_{2}\right\}$; in other words, $\forall(s, v) \in$ $g * \rho_{1}, \exists(s, \Leftrightarrow v) \in \rho_{2}$, and $\forall(s, v) \in \rho_{2}, \exists(s, \Leftrightarrow v) \in g * \rho_{1}$. See Figure $1(\mathrm{~d})$ for an example.

It is easy to verify that these relationships are symmetrical relations. Immediately we can prove that this characterization has exhaustively enumerated all the possible cases between a pair of oriented primitive features. 
Proposition 2.2.3 Distinct, 1-congruent, 2-congruent and complementary are the only possible relationships between a pair of primitive features.

Corollary 2.2.4 Except for a pair of planar surface primitive features, distinct, 1-congruent, 2-congruent and complementary relationships are mutually exclusive relations between a pair of primitive features.

Proposition 2.2.5 If features $F_{1}=\left(S_{1}, \rho_{1}\right), F_{2}=\left(S_{2}, \rho_{2}\right)$ are complementary of each other, where $a\left(S_{1}\right)=S_{2}, a \in \mathcal{E}^{+}$, and $G_{1}, G_{2}$ are the symmetry groups of $F_{1}, F_{2}$ respectively, then $a G_{1} a^{-1}=G_{2}$. In particular, if $S_{1}=S_{2}$ then $G_{1}=G_{2}$ (the necessary condition for surface contact).

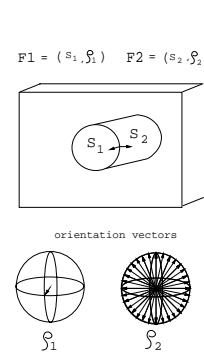

(a) distinct

$$
\mathbf{F} 1=\left(\mathrm{s}_{1}, \rho_{1}\right) \quad \mathbf{F} 2=\left(\mathrm{s}_{2}, \rho_{2}\right)
$$

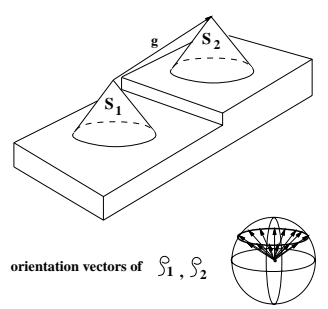

(b) 1-congruent
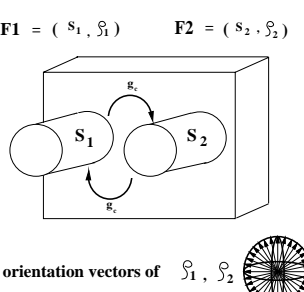

(c) 2-congruent

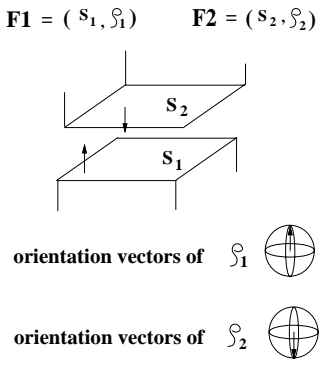

(d) complementary

Fig. 1. Four types of surface relations between a pair of solids.

Symmetry Group of Multiple Oriented Surfaces In the next few propositions we shall explore how the symmetry group of a compound feature is expressed by the symmetry groups of its component primitive features. The first case we consider is when a compound feature $F$ is composed of $n$ pairwise distinct features.

Proposition 2.2.6 Given a compound feature $F=(S, \rho)$ of primitive features $F_{1}=\left(S_{1}, \rho_{1}\right), \ldots, F_{n}=\left(S_{n}, \rho_{n}\right)$ where $F_{1}, \ldots, F_{n}$ are pairwise distinct primitive features with symmetry groups $G_{1}, \ldots G_{n}$ respectively. Then the symmetry group $G$ of $F$ is $G=G_{1} \cap \ldots \cap G_{n}$.

Proposition 2.2.7 Let a compound feature $F=(S, \rho)$ be composed of a pair of primitive features $F_{1}=\left(S_{1}, \rho_{1}\right)$ and $F_{2}=\left(S_{2}, \rho_{2}\right)$ which are 1-congruent of each other. If $G_{1}, G_{2}$ are the symmetry groups of $F_{1}, F_{2}$ respectively, and $G$ is the symmetry group of $F$ then $G=G_{1} \cap G_{2}$.

Proposition 2.2.8 Let a compound feature $F=(S, \rho)$ be composed of a pair of primitive features $F_{1}$ and $F_{2}$ which are 2-congruent of each other via $g_{c}$ (Definition 2.2.2). If $F_{1}=\left(S_{1}, \rho_{1}\right), F_{2}=\left(S_{2}, \rho_{2}\right)$ have symmetry groups $G_{1}, G_{2}$ 
respectively, and $G$ is the symmetry group of $F$ then $G=<g_{c}>\left(G_{1} \cap G_{2}\right)$ where $\left\langle g_{c}\right\rangle$ denotes the subgroup of $\mathcal{E}^{+}$generated by $g_{c}$.

In general, the symmetry group $G$ of a compound feature $F$ can be found from the intersection of the symmetry groups $G_{i}$ of its primitive features. The only exception is when a mapping which flips a pair of 2-congruent features in $F$ is also a member of $G$. These kinds of mappings are new symmetries that do not exist in any $G_{i}$ and the new group they generate is a discrete group.

\subsection{Surface Contact: Computationally Tractable}

As one can observe from the proven results for surface contact, the intersection of symmetry groups of the primitive features is one of the crucial operations in determining the relative motions of contacting solids. We face two computational problems: (1) How to denote symmetry groups, which can be finite, infinite, discrete or continuous, on computers? (2) How to do intersections of subgroups of $\mathcal{E}^{+}$on computers efficiently? We have successfully implemented an efficient $\mathbf{T} \mathbf{R}^{4}$ subgroup intersection algorithm using geometric invariants denotation of the groups [9,10] (Figures 2 and 3 ). The basic symmetry group of each surface of a solid is obtained by a straightforward mapping from the boundary (surface) file of the solid to their respective canonical symmetry groups. The

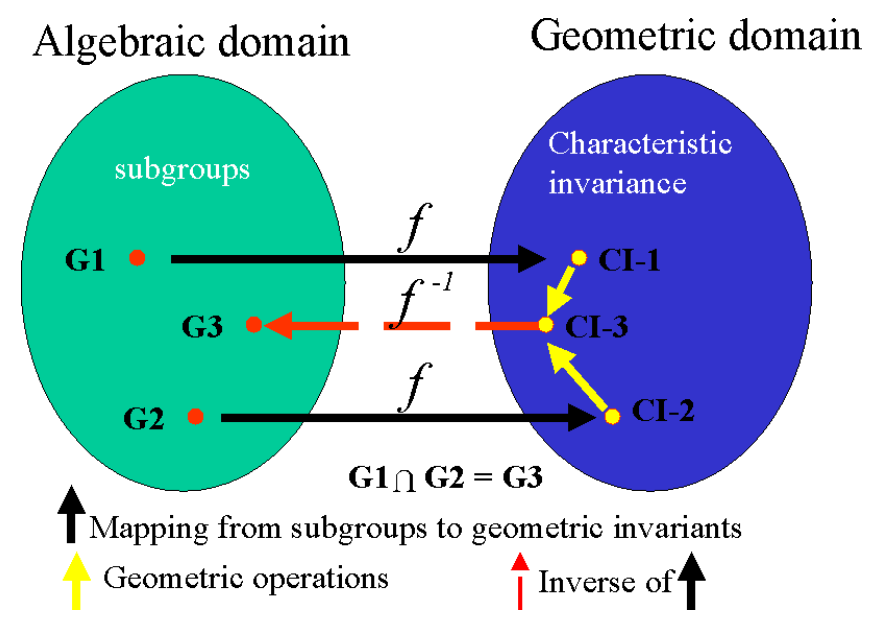

Fig. 2. A geometric representation, characteristic invariants, for the subgroups of the Euclidean group

group theoretical formalization of surface contact has also been embedded into an assembly planning system $K A 3$ (Figure 4).

\footnotetext{
${ }^{4}$ A symmetry group $G$ (a set of motions) that can be divided into a translation subgroup $T$ and a rotation subgroup $R$ - a semidirect product $G=T R$. We call such a group a TRsubgroup of $\mathcal{E}^{+}$.
} 


\section{Group Intersection}
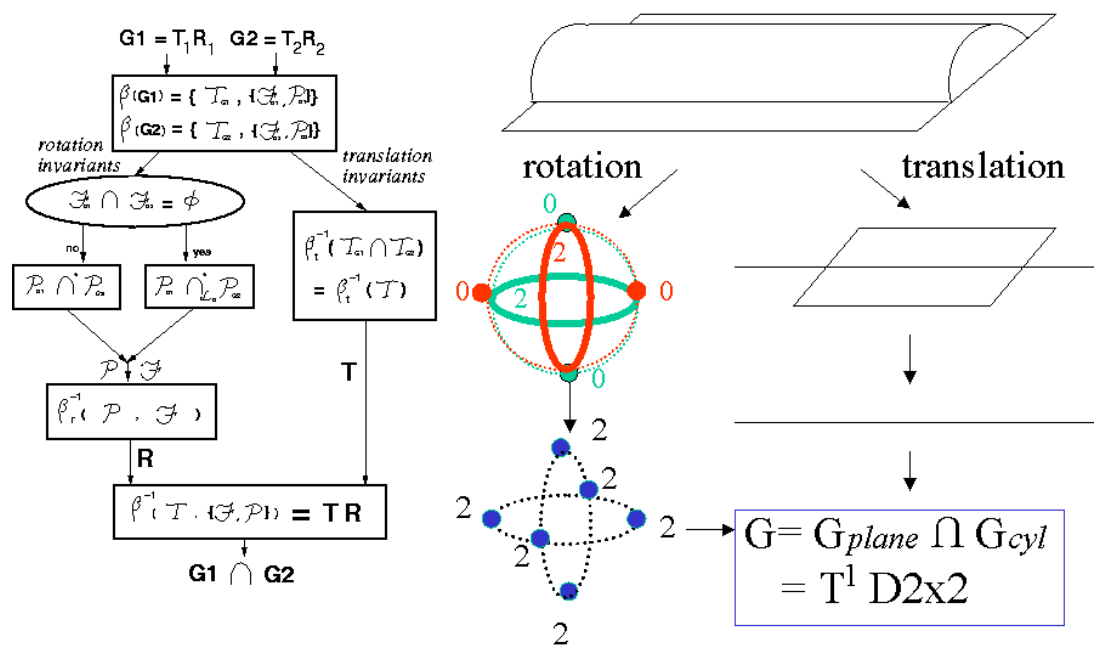

This is an $O\left(\mathrm{n}^{2}\right)$ algorithm, where $\mathrm{n}$ is the number of countable poles.

Fig. 3. Left: TR group intersection algorithm using translation and rotation invariants. Right: an example of symmetry group intersection (symmetry groups of planar and cylindrical surfaces)

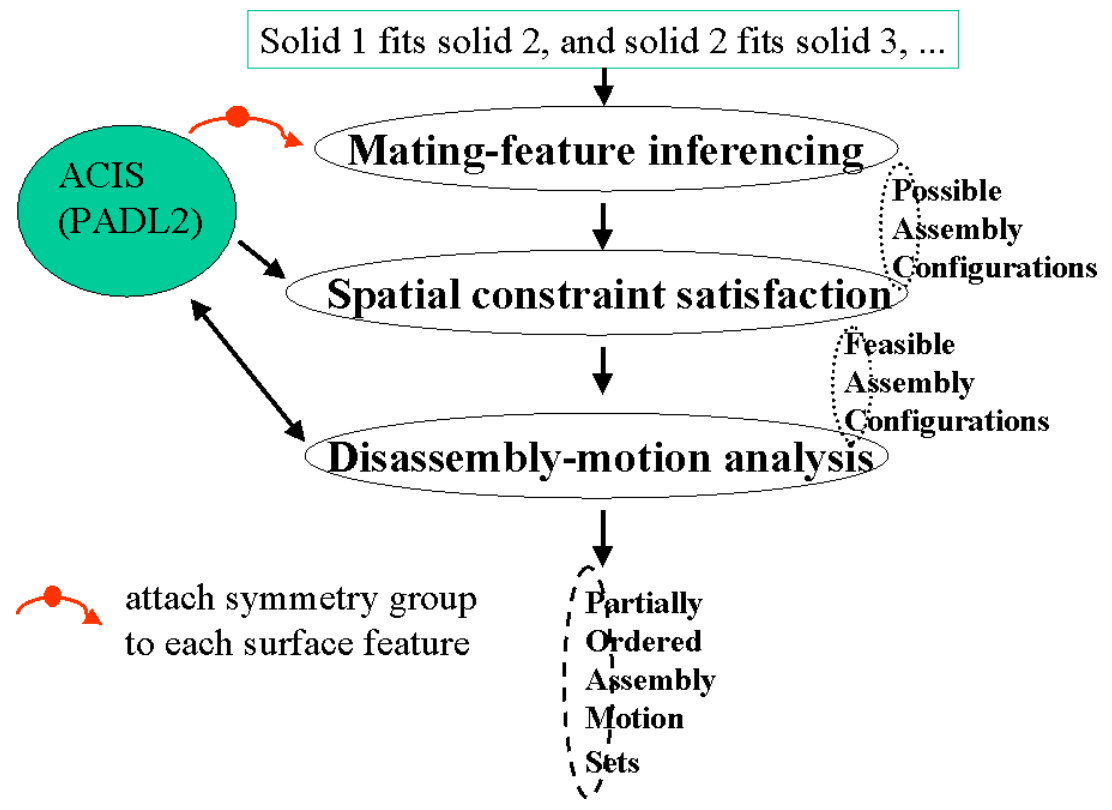

Fig. 4. An assembly planner $K A 3$ takes high-level task specifications and generates robot executable assembly instructions. 


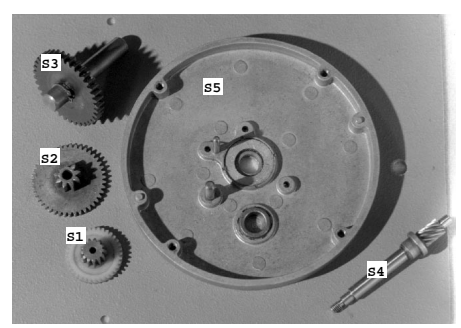

(A)

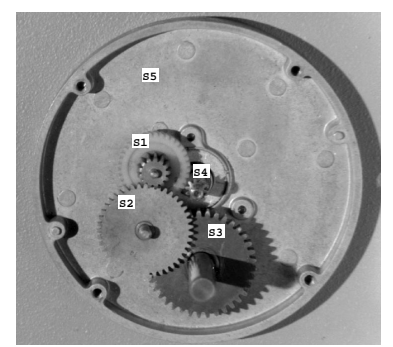

(B)

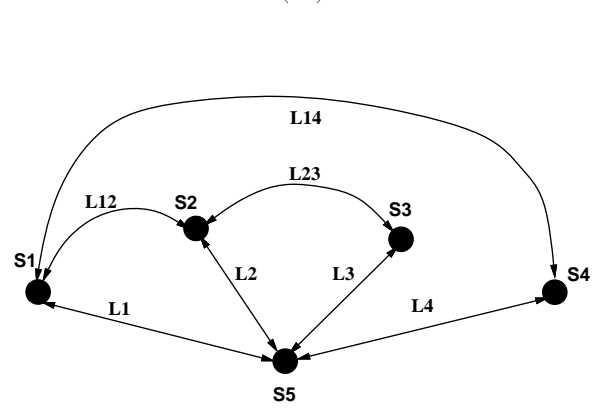

(C)

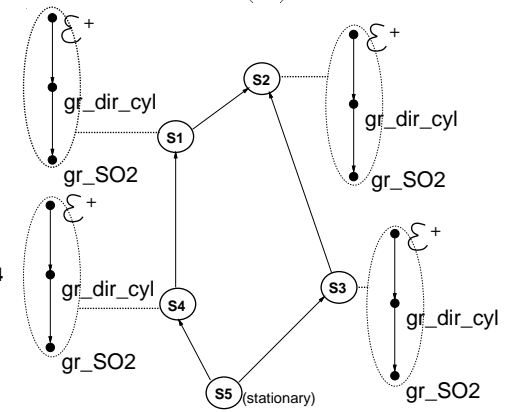

(D)

Fig. 5. (A) A five-part gearbox assembly. (B) The top view of the gearbox configuration automatically computed by $K A 3$. (C) Representation of the gearbox assembly in terms of contacting compound feature symmetry groups, see text for details. (D) The output POAMS for the gearbox assembly. Each solid $S_{i}$ is associated with a sequence of motions in terms of the symmetry group of its contacting surfaces with the rest of the assembly. Notice, during disassembly each solid is going through a 2-surface contact $\left(S O(2)\right.$, assembled configuration), 1-surface contact $\left(\mathcal{G}_{\text {dir_cyl }}\right)$ and finally, no contact $\left(\mathcal{E}^{+}\right.$, disassembled configuration).

As an example of assembly specification using symmetry groups, see Figure 5 for a five-part gearbox. The representation of the assembly is shown in Figure $5(\mathrm{C})$, where $L_{i}, i=1 . .4$ is the symmetry group of the contacting compound feature between solids $S_{i}$ and $S_{5} . L_{i}=a_{i} S O(2) a_{i}^{-1}, i=1 . .4, S O(2)$ is a one degree rotation group resulted from the intersection of the symmetry group of a plane with that of a cylinder (the compound feature composed of two surfaces of the shaft of a gear). $L_{i j}=L_{i} L_{j}=a_{i j} S O(2) b_{i j} S O(2) c_{i j}, i, j=1 . .4$ indicates that the relative positions between gears (non-surface contact) are simply determined by rotations in $S O(2)$ and some specific translations $a_{i j}, b_{i j}, c_{i j}$, where the relative gear pitch ratio is also embedded. This representation of the gearbox (Figure $5(\mathrm{C})$ ) specifies precisely the articulated gearbox assembly. After a disassembly analysis by moving one solid away from this contacting network (Figure $5(\mathrm{C})$ ) at a time, a partially ordered assembly motion set (POAMS) is found (Figure 5 (D)). 


\section{General Contact and Computational Issues}

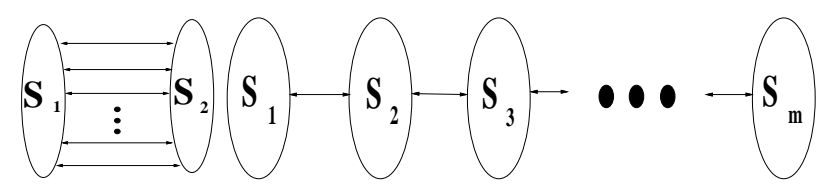

(A)

Fig. 6. (A) Solids $S_{1}$ and $S_{2}$ have $n$ parallel contacts. (B) Solids $S_{1}, S_{2}, \ldots S_{m}$ form a sequential contact chain

We have divided the general contact motion among solids into surface contact (lower pairs), which we have successfully treated computationally, and the nonsurface-contact (higher pairs). They have the following specific forms:

1. Two solids have $n$ surface contact, the relative position of solid 2 with respect to solid 1:

$$
l_{1}^{-1} l_{2} \in f_{1} G f_{2}^{-1}
$$

where $G$ is the symmetry group of the compound feature composed of all the contact primitive features of $S_{1}$ or $S_{2}$. If $F$ is composed of $n$ pairwise distinct features $F_{i}$, then $G=\cap G_{i}$, where $G_{i}$ is the symmetry group of $F_{i}$.

2. Two solids have $n$ general contact (Figure $6(\mathrm{~A})$ ), the relative position of solid 2 with respect to solid 1:

$$
l_{1}^{-1} l_{2} \in f_{11} G_{11} \sigma_{1} G_{21} f_{21}^{-1} \cap f_{12} G_{12} \sigma_{2} G_{22} f_{22}^{-1} \cap \ldots \cap f_{1 n} G_{1 n} \sigma_{n} G_{2 n} f_{2 n}^{-1}
$$

where $G_{i j}$ is the symmetry group of primitive feature $j$ of $S_{i}$ and $f_{i j}$ is its feature coordinates.

3. $m$ solids have a chaining general contact (Figure $6(\mathrm{~B})$ ), the relative location of solid $m$ with respect to solid 1:

$$
l_{1}^{-1} l_{m} \in f_{1} G_{12} \sigma_{1} G_{21} f_{21}^{-1} f_{2} G_{23} \sigma_{2} G_{32} f_{32}^{-1} \ldots f_{m-1} G_{(m-1) m} \sigma_{m-1} G_{m(m-1)} f_{m(m-1)}^{-1}
$$

where $G_{i j}$ is the symmetry group of the surface on solid $i$ in contact with solid $j$.

Encouraged by our existing work on making the group theoretical formalization of surface contact (case 1. above) tractable, our goal is to seek the computational means to deal with the general contact using group theoretical formalization (cases 2. and 3.).

Many open problems remain: (1) Can the geometric representation used for TR groups be used for group product? It is known that in general, a product of groups is not a group. For TR groups, the TR-restriction presented in [9, 
10] provides us with a constructive way to judge from its invariants whether a product remains a TR group. However we do not yet know how to compute a product from the invariants of its subgroups. (2) Given an algebraic surface, how can one find its symmetry group computationally? We intend to investigate the possibility of using the Gröbner basis of a given polynomial to find its symmetry group. An alternative is to use semi-algebraic set to represent the contacting surfaces and find the continuous symmetries using Lie algebra ${ }^{5}$, but under this formalism it is not clear how to deal with discrete symmetries effectively. (3) Given a set of $n$ algebraic surfaces and their respective symmetry groups, what is the exact algorithm to find the symmetry group for the whole set? [9-11] have only proved results for subcases, i.e. $n$ distinct surfaces, a pair of 1-cong or 2 -cong surfaces. No proven result for the most general case yet exists. We are going to further study those compound features with more complicated inner structures. For example, one may define a concept of $n$-congruence on $n$ features $F_{1} \ldots F_{n}$ as requiring that there exists $g \in \mathcal{E}^{+}$such that $g\left(F_{i}\right)=F_{(i \bmod n)+1}$; this is a natural extension of 2-congruence. Such congruences will give rise to new symmetries of the compound feature.

\section{Conclusion}

This work provides a good example of applying algebra to solve a fundamental problem in robotics: solids in contact. It reflects both the power of group theory, and the effort one has to expend to make a mathematical theory computationally feasible. We establish a group theoretical formalization of general contact motion. The generality of this approach allows the treatment of solids in contact as subgroup manipulations, and provides a uniform computational platform for both continuous and discrete groups. The next challenge is to construct tools enabling computations of higher pairs, which will make this work computationally complete. Our previous work has shed some light on the feasibility of achieving this goal.

\section{References}

1. M.P. Do Carmo. Differential Geometry of Curves and Surfaces. Prentice Hall, New Jersey, 1976.

2. I. Drori, L. Joskowicz, and E. Sacks. Contact analysis of spatial fixed-axes pairs using configuration spaces. In IEEE International Conference on Robotics and Automation, pages 578,584. IEEE, 1999 .

3. J.M. Hervé. Analyse structurelle des mécanismes par groupe des déplacements. Mechanism and Machine Theory, 13(4):437 - 450, 1977.

4. L.S. Homem de Mello. Task Sequence Planning for Robotic Assembly. PhD thesis, Carnegie Mellon University, 1989.

5. L. Joskowicz and E. Sacks. Computer-aided mechanical design using configuration spaces. IEEE Computers in Science and Engineering, page 14/21, Nov/Dec 1999.

\footnotetext{
${ }^{5}$ Thanks to an anonymous reviewer for pointing this out
} 
6. L. Joskowicz, E. Sacks, and V. Kumar. Selecting an effective task-specific contact analysis algorithm. IEEE Workshop on New Directions in Contact Analysis and Simulation, 1998.

7. J.C. Latombe. Robot Motion Planning. Kluwer, 1993.

8. C. Laugier. Planning fine motion strategies by reasoning in the contact space. In IEEE International Conference on Robotics and Automation, pages 653-661, Washington, DC, 1989. IEEE Computer Society Press.

9. Y. Liu. Symmetry Groups in Robotic Assembly Planning. PhD thesis, University of Massachusetts, Amherst, MA., September 1990.

10. Y. Liu. A Geometric Approach for Denoting and Intersecting TR Subgroups of the Euclidean Group. DIMACS Technical Report, Rutgers University, 93-82:1-52, 1993.

11. Y. Liu and R. Popplestone. A Group Theoretical Formalization of Surface Contact. International Journal of Robotics Research, 13(2):148 - 161, April 1994.

12. Y. Liu and R.J. Popplestone. Assembly planning from solid models. In IEEE International Conference on Robotics and Automation, Washington, DC, 1989. IEEE Computer Society Press.

13. Y. Liu and R.J. Popplestone. Symmetry constraint inference in assembly planning. In Eighth National Conference on Artificial Intelligence, Boston, Mass., July/August 1990.

14. Y. Liu and R.J. Popplestone. Symmetry groups in analysis of assembly kinematics. In IEEE International Conference on Robotics and Automation, Washington, DC, May 1991. IEEE Computer Society Press.

15. Y. Liu and R.J. Popplestone. Symmetry groups in solid model-based assembly planning. In Artificial Intelligence Applications in Manufacturing, pages 103,131, Cambridge,Massachusetts, 1992. AAAI Press and The MIT Press.

16. T. Lozano-Pérez, J.L. Jones, E. Mazer, P.A. O’Donnell, W.E.L. Grimson, P. Tournassoud, and A. Lanusse. Handey: A robot system that recognizes, plans, and manipulates. In IEEE International Conference on Robotics and Automation, pages 843-849, Washington, DC, March 1987. IEEE Computer Society Press.

17. A.T. Miller and P.K. Allen. Examples of 3d grasp quality computations. In IEEE International Conference on Robotics and Automation, pages 1240,1246. IEEE, 1999.

18. David Montana. The kinematics of contact and grasp. The International Journal of Robotics Research, 7(3):17-32, Jume 1988.

19. R.J. Popplestone. Group theory and robotics. In M. Brady and R. Paul, editors, Robotics Research, The First Int. Symp. MIT Press, Cambridge,Massachusetts, 1984 .

20. R.J. Popplestone, A.P. Ambler, and I. Bellos. An interpreter for a language for describing assemblies. Artificial Intelligence, 14(1):79-107, 1980.

21. E. Sacks. Practical sliced configuration spaces for curved planar pairs. Internaltional Journal of Robotics Research, 18(1):59-63, January 1999.

22. C.A. Tanaglia, D.E. Orin, R.A. LaFarge, and C. Lewis. Toward development of a generalized contact algorithm for polyhedral objects. In IEEE International Conference on Robotics and Automation, pages 2887,2892. IEEE, 1999.

23. F. Thomas and C. Torras. Inferring feasible assemblies from spatial constraints. IEEE Transactions on Robotics and Automation, 8(2):228,239, April 1992.

24. Randall Wilson and Jean-Claude Latombe. Geometric reasoning about mechanical assembly. Artificial Intelligence, 71(2), December 1994.

25. J.D. Wolter. On the Automatic Generation of Plans for Mechanical Assembly. $\mathrm{PhD}$ thesis, University of Michigan, 1988. 